
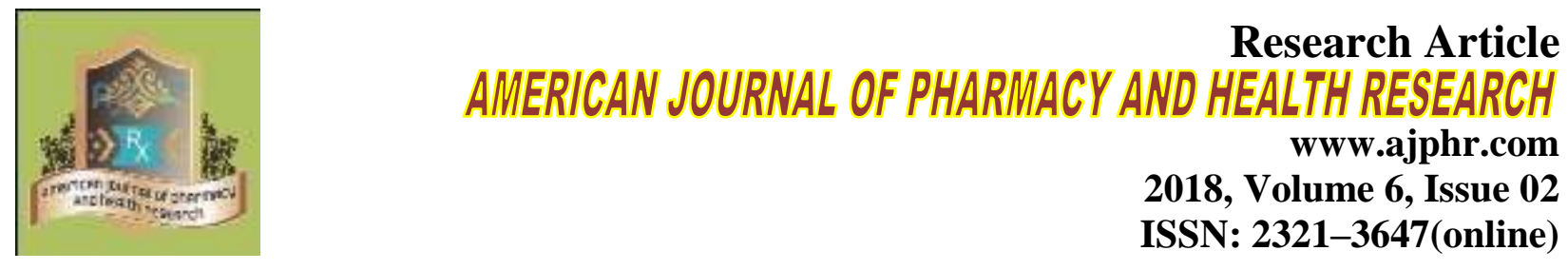

\title{
Comparison of Outcomes of Two Different Treatment Methods In The Management of Diabetic Foot Ulcer
}

\author{
M.V.R. Harsha Vardhan ${ }^{1}$, Y. Suraj Varma ${ }^{1}$, S. Parimalakrishnan ${ }^{1}$, Junior Sundresh*2 \\ 1.Department Of Pharmacy, Annamalai University \\ 2.Deputy Medical Superintendent, Rajah Muthiah Medical College \& Hospital, Annamalai \\ University
}

\begin{abstract}
The aim of this study was to compare the outcomes of surgical versus non- surgical treatment in the management of Diabetic Foot Ulcer. A Prospective Observational Study which was carried out in the Department of Surgery of Rajah Muthiah Medical College Hospital a tertiary health care hospital in Chidambaram, Cuddalore District, Tamilnadu in the period of November 2016 to April, 2017 (6 months). The patient demographic, disease, ulcer size, drugs and treatment prescribed were collected in a specially designed data collection form which also included bacterial culture tests. Upon enrolment for the study 65\% (39) of the patients diagnosed for diabetic foot were male and $35 \%$ (21) were female. The demographic classification suggests that diabetic foot incidence has increased upon ages of people ranging between the mean ages of $45+/-5$ years to $60+/-5$ years. The bacterial distribution over the patients showed that, out of which Escherichia coli (28.3\%) and Staphylococcus aureus (15\%) are the most common bacteria. The outcomes mainly depend upon the grading of infection and ulcer size. The superficial ulcers can be treated more effectively with antibiotics and regular dressings, whereas the deep ulcers and gangrene should be intervened with surgical procedures. The assessed study reports that clinical pharmacist intervention by patient education and medication counselling creates a significant influence in medication adherence
\end{abstract}

Keywords: Diabetic foot ulcer, surgical treatment, Non-surgical treatment, management 


\section{INTRODUCTION}

Diabetic foot ulcers and infections are responsible for $>30 \%$ of the hospitalizations related to diabetes mellitus. $25 \%$ of people with diabetes mellitus are estimated to develop a foot ulcer during their lifetime. Diabetic foot ulceration is also an expensive complication of diabetes mellitus, owing to both medical care and on account of time lost from work and loss of income and financial independence. The majority (>80\%) of foot ulcers in India arise in neuropathic feet, with only a third having vascular insufficiency, which, importantly, implies that most of these ulcers can be prevented with proper patient education on appropriate foot care. The management of diabetic foot disease may seem poorly defined by comparison with complications such as nephropathy, hyper-lipidaemia and retinopathy, for which clear guidelines exist. A multidisciplinary team, approach, particularly in specific diabetic foot clinics, is very successful in avoiding and treating foot complications, This strategy has been shown to reduce both the incidence of leg amputations (by $40 \%$ or greater) and the duration of in-patient admissions for diabetic foot treatment . Foot ulceration is common, affecting up to $25 \%$ of patients with diabetes during their lifetime. About $85 \%$ of leg amputations were preceded by foot ulcers and Diabetes remains a major cause of non-traumatic amputations worldwide by rates being as much as 15 times higher than in the non-diabetic population. Prevention is the first step towards solving diabetic foot problems. Although it was estimated that an ankle is lost to diabetes somewhere in the world every 30 seconds, a more important fact is that up to $85 \%$ of all amputations in diabetes should be preventable. Strategies aimed at preventing foot ulcers are cost-effective and can even be cost-saving if increase education and effort are focused on those patients with recognized risk factors for the development of foot problem. So in this study, an attempt was made to examine the various stages of diabetic foot ulcer, treatment procedures and the impact of diabetic foot ulcer in the quality of life of the patients who had undergone the therapy.

\section{Aims and Objectives:}

The aim of the study was to compare the outcomes of surgical versus non- surgical treatment in the management of Diabetic Foot Ulcer and the objectives were to find the organism pattern, pattern of antibiotics prescribed and to compare the outcome of non-surgical and surgical treatment in patients with complicated diabetic peripheral neuropathy, healing time between the two groups and assess the quality of life in patients with diabetic foot using standard questionnaire. 


\section{MATERIALS AND METHOD}

A prospective observational study was carried out between the period of November 2016 to April 2017 (6 months) in Rajah Muthiah Medical College Hospital, Annamalai University, Chidambaram, Cuddalore district, Tamilnadu. 74 Patients diagnosed with Diabetic foot ulcer were treated in the hospital out of which 60 patients complied with the study and patient consents were collected. The patients were included based upon the suitable inclusion and exclusion criteria. Demographic data, length of hospital stay, diagnosis, medication chart and other relevant details were obtained from the patient case record documents. The WAGNER scale was used to classify the ulcers based upon the size and site of diabetic foot ulcer. The treatments of the ulcers were based upon the grading based upon the scale. Various domains were assessed for studying quality of life of patients using WHO- BREF questionnaire and analysed for pre-counselling and post-counselling outcomes.

\section{RESULTS AND DISCUSSION}

Applying inclusion and exclusion criteria a total of 60 samples were enrolled into the study. Out of which 39(65\%) were male and 21 (35\%) were female (fig 1).Based on age the patients were categorized into 5 groups, which consisted of people ranging above 30-40 years in which 2 were female (3.3\%) and people between age groups of 51-60 years had a highest incidence ( 30\% )of diabetic foot ulcer in our study (fig 2).Diabetic patients with personal history of smoking, alcoholism or tobacco had an increased risk of developing complications; as they would affect the wound healing time either directly or indirectly. The following data assessed the percentage of people with habits of smoking and alcoholism (fig 3). The conservative group had the lowest days of hospital stay, this may be due to various factors such as the ulcer site, size, extent of infection etc., the patients who have not been completely healed or condition was not brought under control were opted to perform surgical intervention to heal the ulcers (fig 4). Superficial ulcers with areas ranging between 1-10sq cm were more reported in conservative groups and as well as surgical groups early management of diabetic foot is important to control the wound and for providing better care (fig 5). The ulcers reported in the study mostly had intact skin and also superficial in nature, they were ranging from $43 \%$ and $21.6 \%$ respectively of all the ulcers. They were graded by using WAGNER method of classification (fig 6).Bacterial culture reports were assessed to find the distribution of bacteria in the patients suffering from diabetic foot ulcers, out of which Escherichia coli (28.3\%) and Staphylococcus aureus (15\%) are the most common bacteria to be found (fig 7). From the reported cases the most common co existing conditions 
were found to be Hypertension, followed by Coronary Artery Disease and lower extremity disease such as Peripheral vascular disease(fig 8). The prescription of medications for diabetes and the bacterial infections is more in conservative group when compared to the surgical group(fig 9).The incidence of the foot ulcers is more in right leg, many factors played role in the occurrence of ulcers they may be due to daily activities, external trauma, poor foot care, wet feet and other health related factors. An unpaired t-test was used to analyse the significant difference between the pre-counselling and post counselling mean scores of physical, psychological, social and environmental factors (table 1). The quality of life was assessed between the two groups; the readings were taken before and after the completion of therapy. the patients reported better quality of life later the therapy, there was a slight decrease in the QOL in surgical group this may have been due to amputation and other conditions due to which the patients have lost a limb and they cannot get back to their lives as it was used to be (table 2).

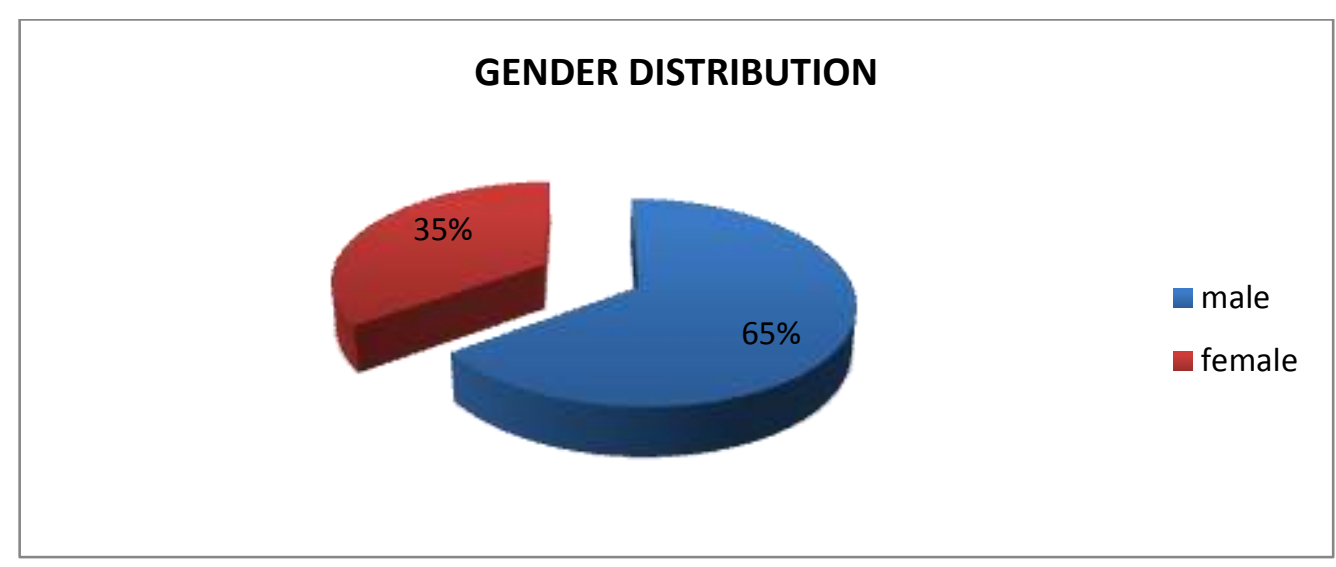

\section{Gender wise Distribution}

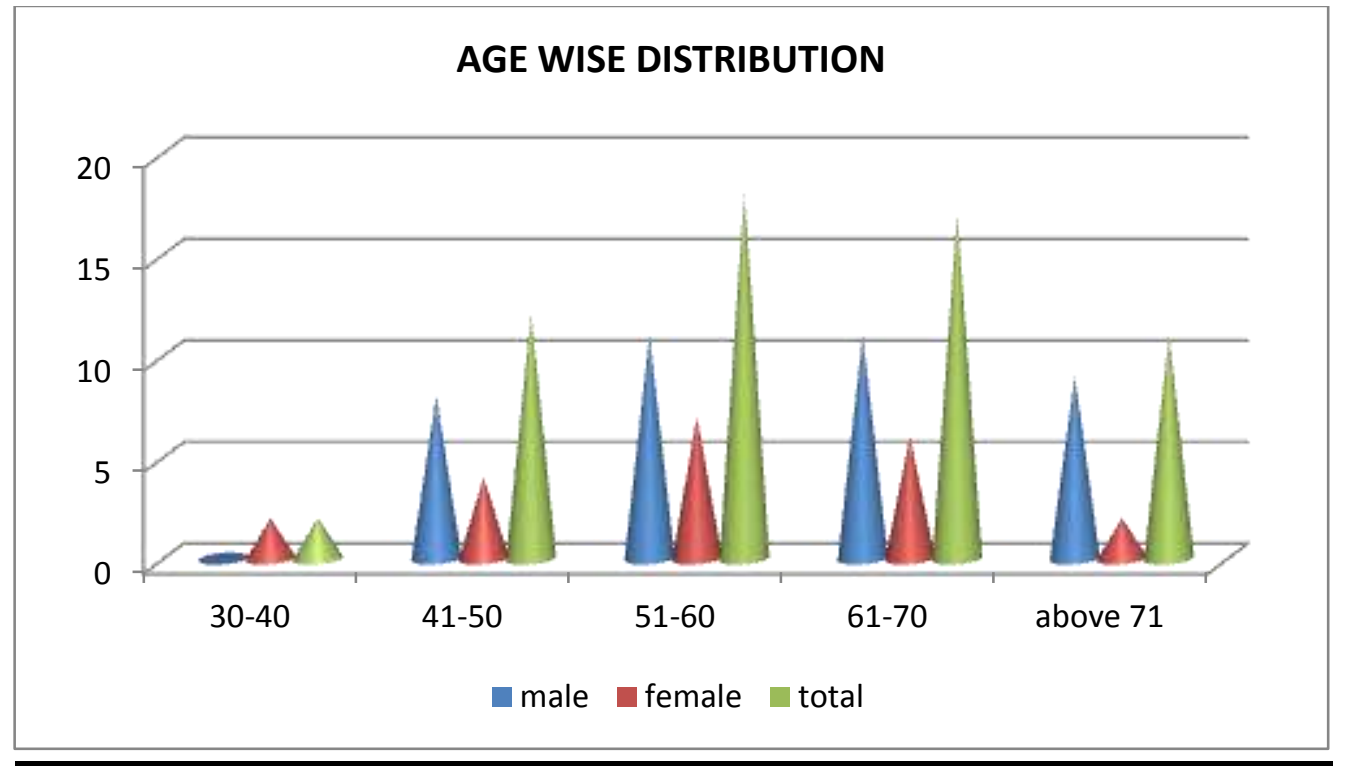

2 Age wise distribution 


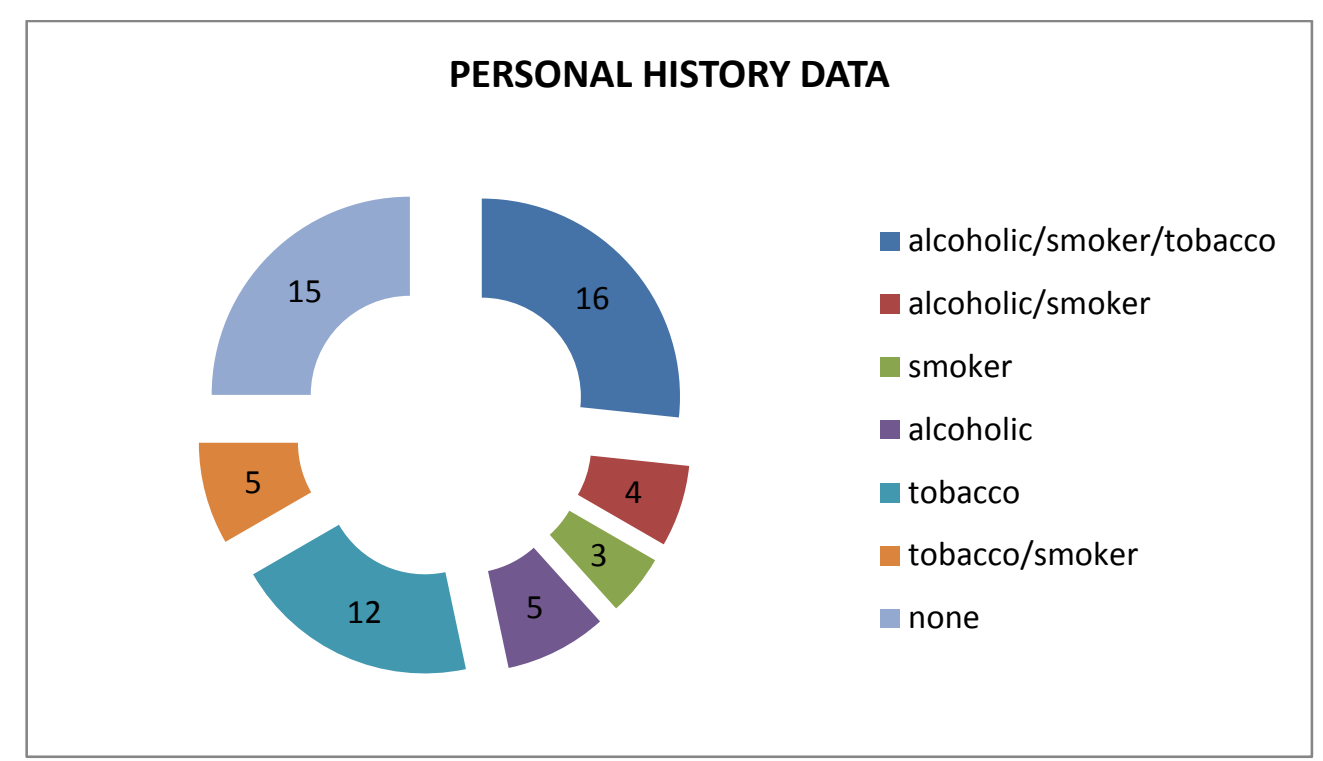

3 Personal history data of patients

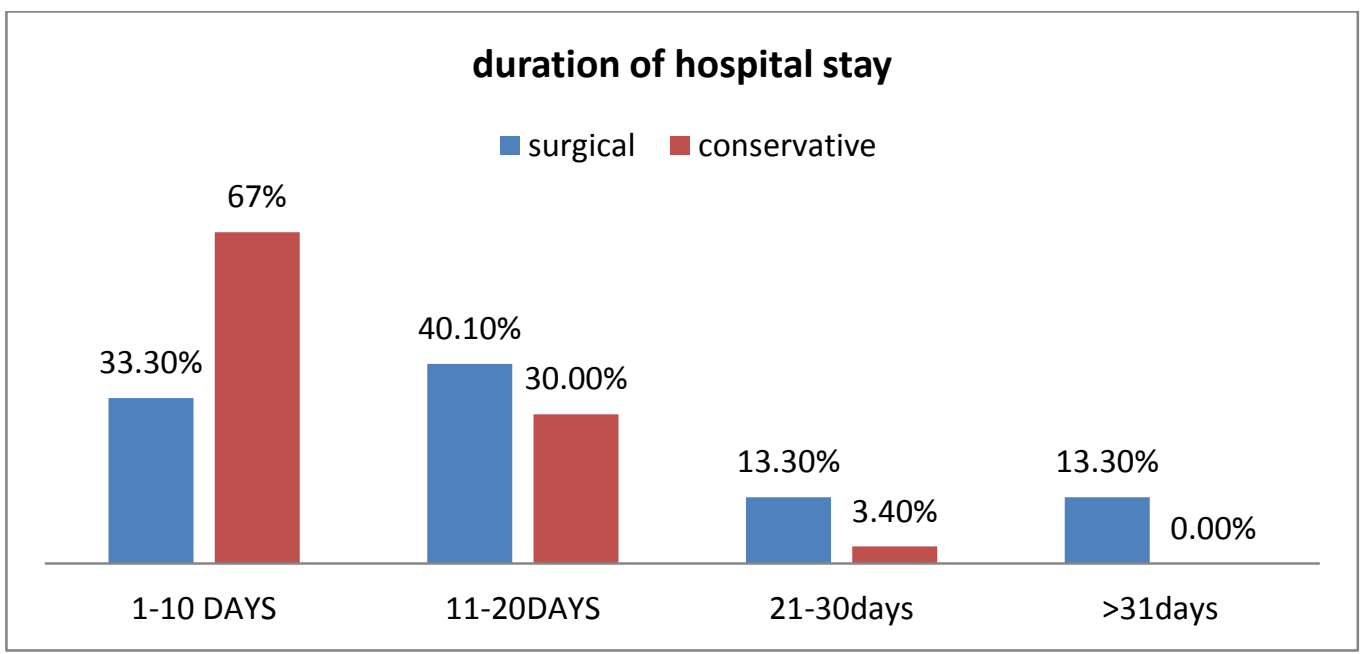

4 Average duration of hospital stay

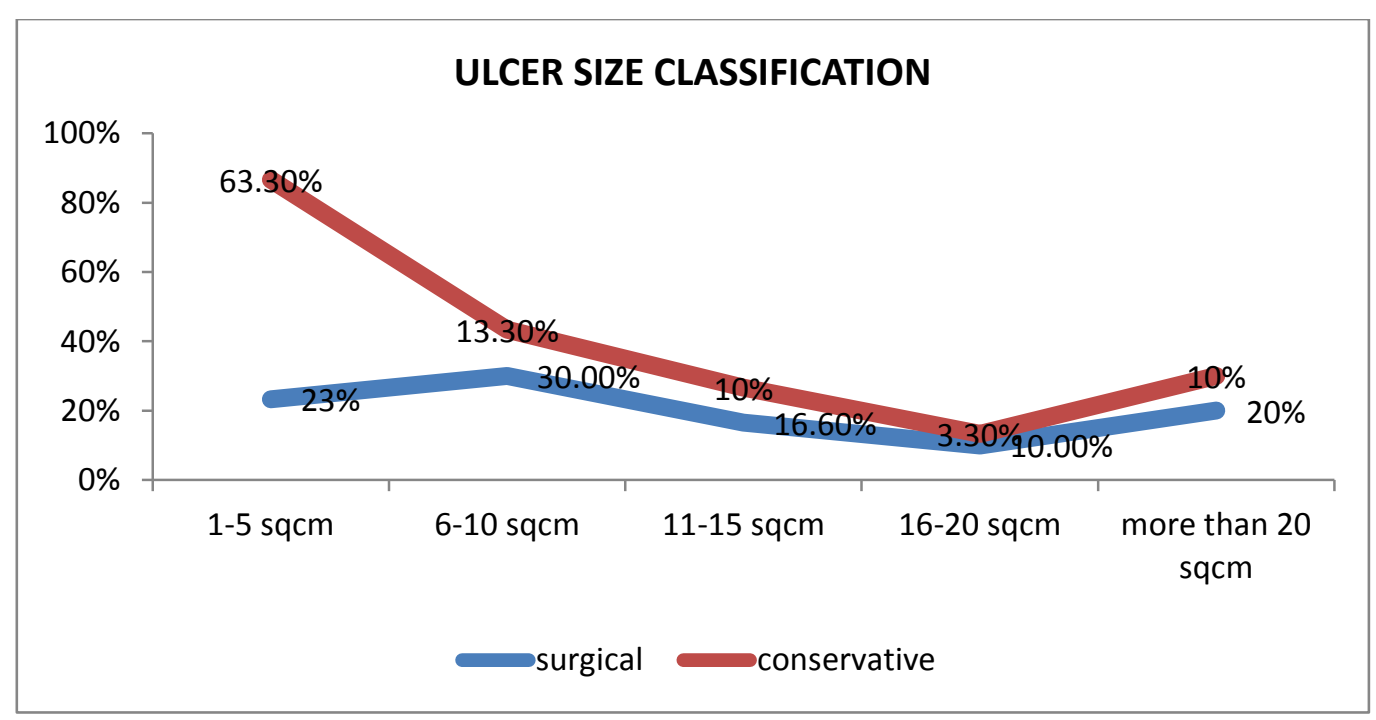

5 Ulcer size classification 


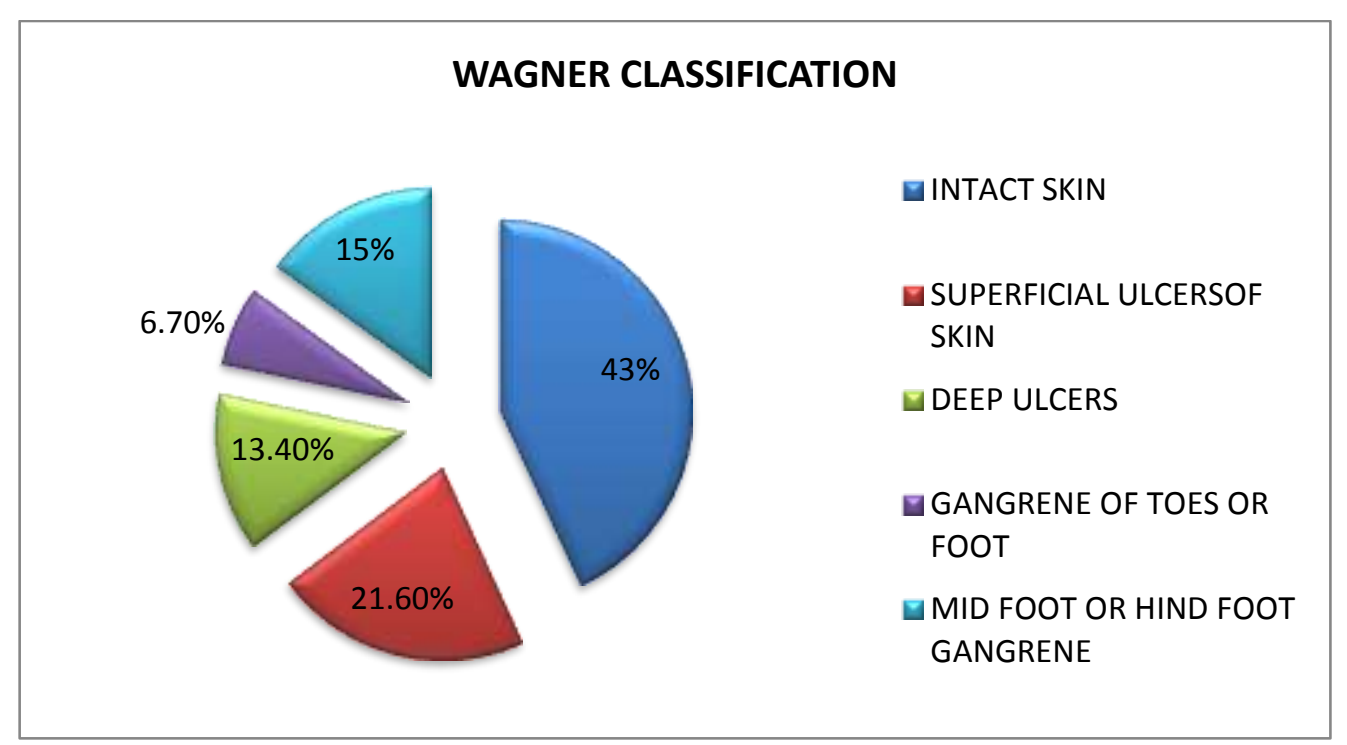

6 Wagner classifications

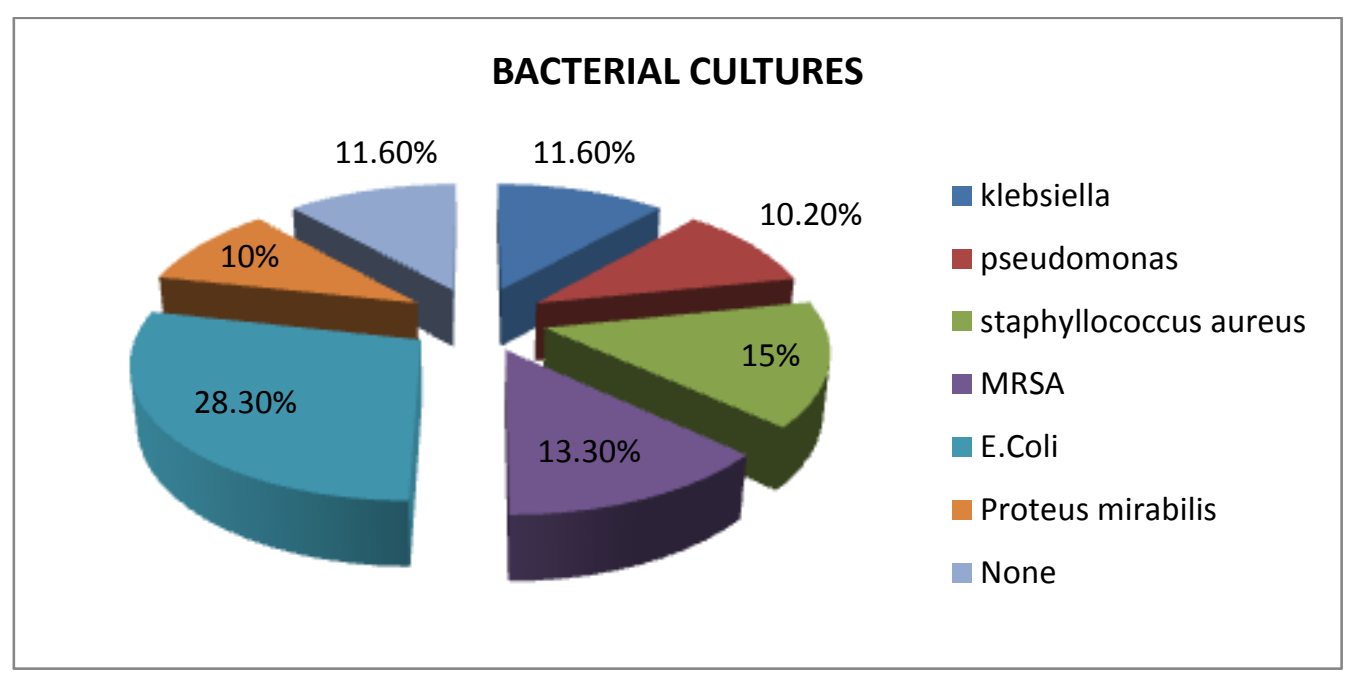

7 Bacterial cultures reported

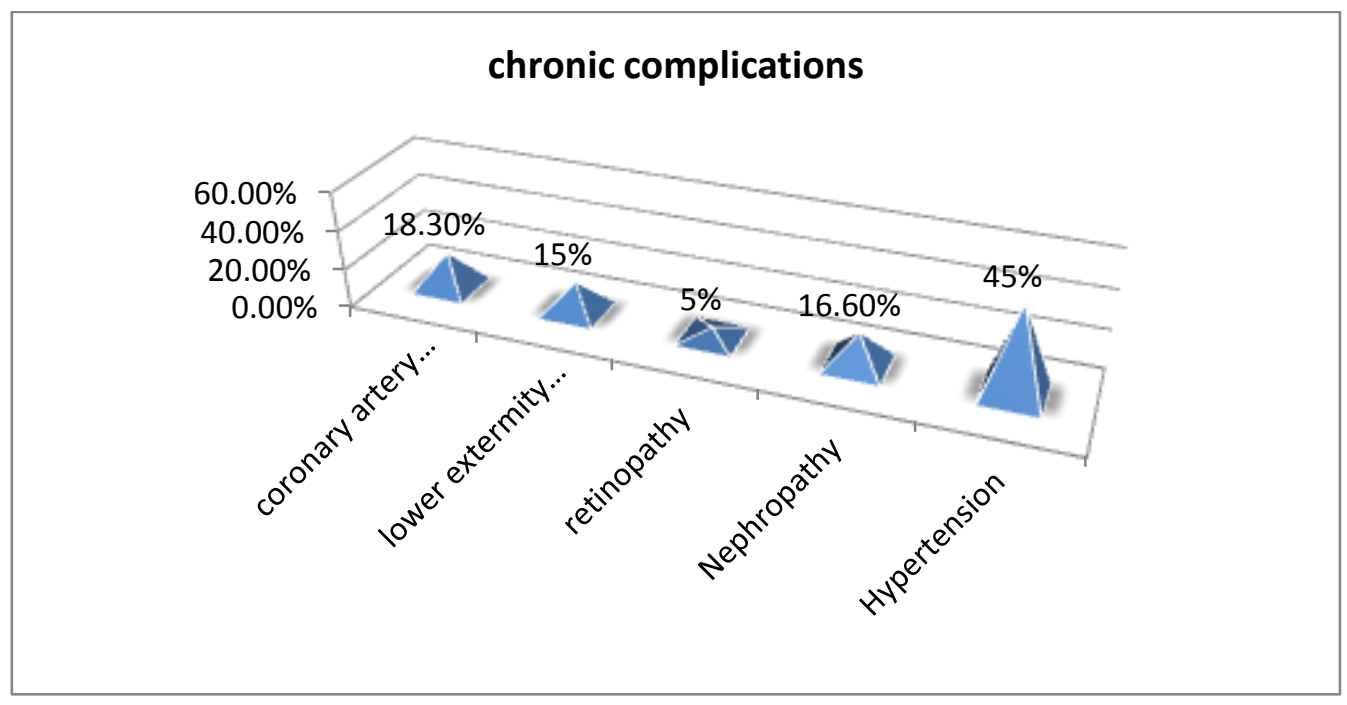

8 Co-Morbid Conditions in the Patients with Diabetic foot 


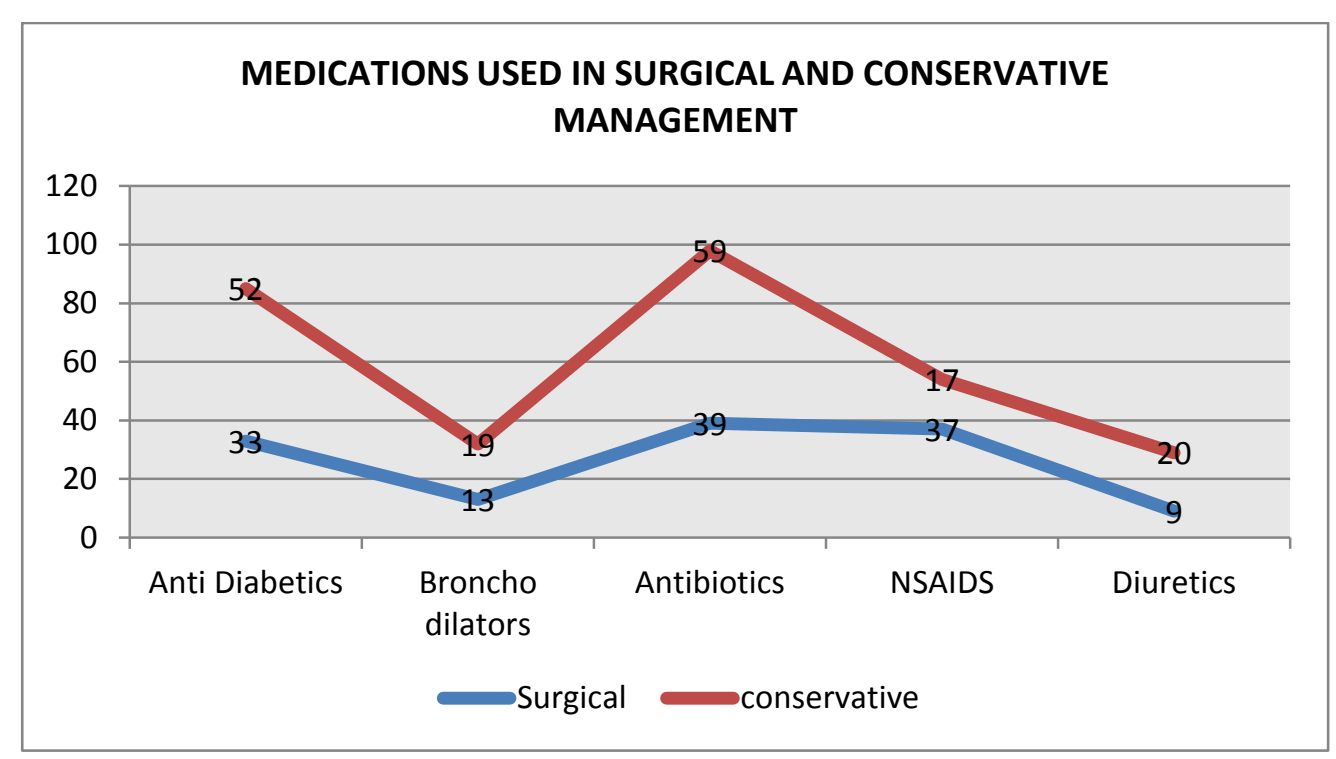

9 Medications used in surgical and conservative management

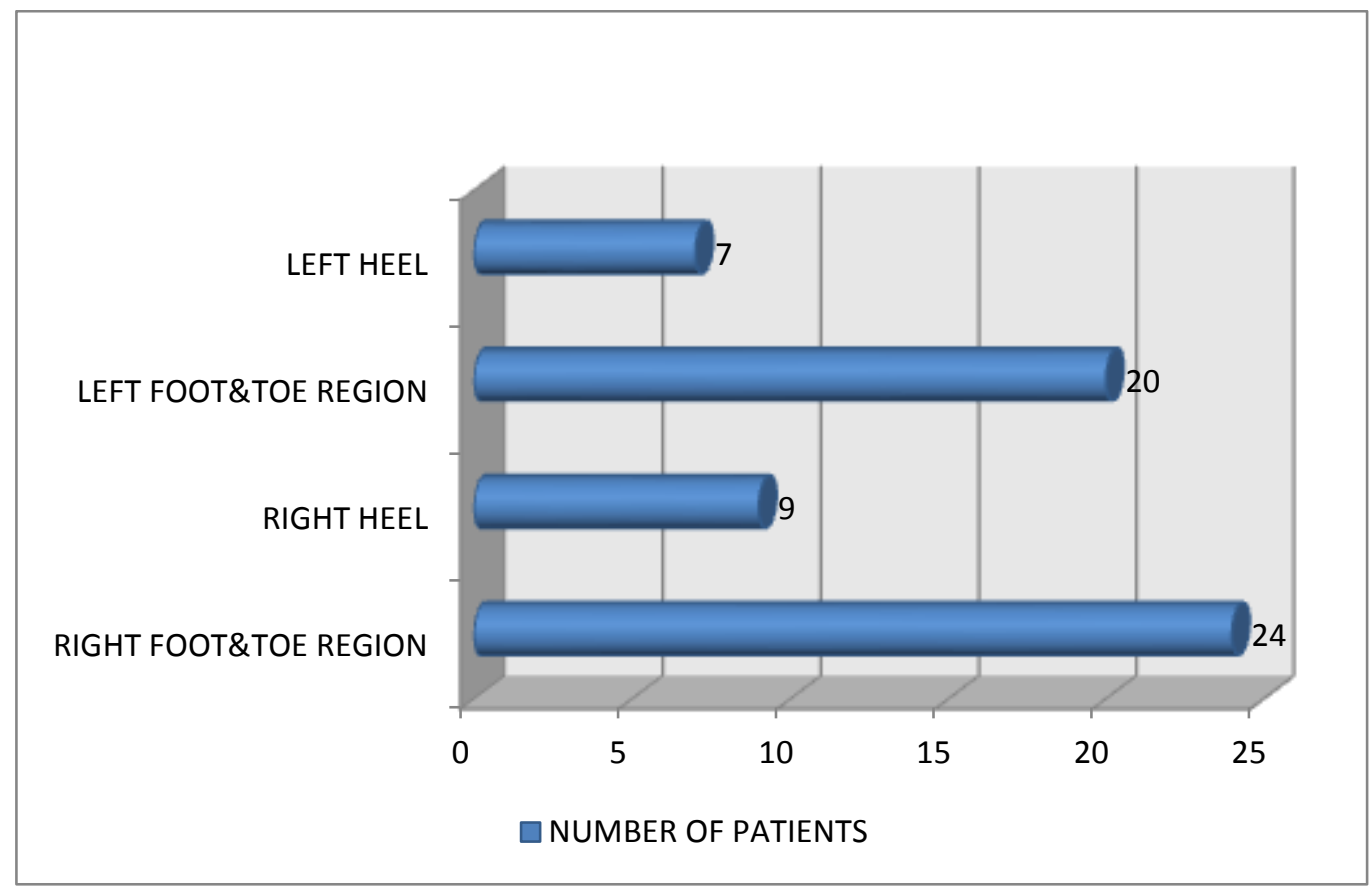

10 Location of foot ulcers

Table 1

\begin{tabular}{llll}
\hline Domains & t Value & P Value & Significance \\
\hline Physical & 3.919 & 1.000 & YES \\
Physiological & 5.014 & 1.000 & YES \\
Social & 3.642 & 1.000 & YES \\
Environmental & 3.980 & 0.958 & YES \\
\hline
\end{tabular}

*Significance was found by comparing the calculating $\mathrm{t}$ value with tabulated $\mathrm{t}$ value at $95 \%$ confidence interval $(\mathrm{p}=0.001)$. 
Table 2

\begin{tabular}{lll}
\hline & $\begin{array}{l}\text { MEAN } \pm \text { SEM } \\
\text { Pre-counselling }\end{array}$ & Post Counselling \\
\hline Physical component & $49.108 \pm 1.804$ & $59.108 \pm 1.804$ \\
Physiological component & $45.365 \pm 1.410$ & $55.365 \pm 1.410$ \\
Social component & $59.054 \pm 1.941$ & $69.054 \pm 1.941$ \\
Environmental component & $52.365 \pm 1.721$ & $62.122 \pm 1.746$ \\
\hline
\end{tabular}

\section{CONCLUSION:}

The study was to compare the treatment of diabetic foot ulcers with antibiotic therapy exclusively to treatment with surgery followed by a short post-operative period of antibiotic therapy. The conservative group received daily wound dressings and therapy of a combination of antibiotics, anti-diabetics and anti-infective to assist the healing of ulcers. The surgical groups were treated with surgical procedures such as skin grafts, Ray's amputation method for removal of damaged tissue and bone segments, followed by antibiotic therapy. The diagnosis of DFU were established based on the combination of physical examination, microbiological culture tests for organisms, assessment of site and size of ulcers with the help of WAGNER grading system of DFU and Diabetic Foot Ulcer scale (DFS). The treatment strategies were adopted from the standard management guidelines of diabetic foot ulcer and other sources. The study showed that the male population is more affected with DFU than female as there were many factors to consider such as daily activities, severity of diabetes, management of wounds and practice of proper foot care. The results suggest that the DFU occurrence was more in people of age ranging between 41-60 years and older which suggests that proper care is necessary for people with greater age. The people with personal history of smoking, alcohol, and on irregular anti-diabetic therapy, had a greater chance of developing diabetic foot complications and healing time was also affected. In conclusion, the comparison of surgical and conservative management of diabetic foot; the outcome mainly depends upon the grading of infection and ulcer size. The superficial ulcers can be treated more effectively with antibiotics and regular dressings, whereas the deep ulcers and gangrene should be intervened with surgical procedures.

\section{LIMITATIONS OF THE STUDY:}

The number of patients obtained and the study duration were limited. If the study was conducted for longer duration, more significant results in all outcomes may have been obtained. The study was limited to inpatients, if the study was extended for outpatients more significant results would have been obtained. 


\section{REFERENCES}

1. Edmonds M, Foster A (2011) the diabetic foot. In: Wass AHJ, Stewart MP (eds) Endocrinology and diabetes. 2nd ed. Oxford University Press, New York.

2. Polikandrioti M, Kalogianni A (2009) Education on foot care in people with diabetes mellitus, type II. Rostrum of Asclepius 8: 107-119.

3. Tennvall GR, Apelqvist J (2000) Health-related quality of life in patients with diabetes mellitus and foot ulcers. J Diabetes Complications 14: 235-241.

4. Abetz L, Sutton M, Brady L, McNulty P, Gagnon DD (2002) The Diabetic Foot Ulcer Scale (DFS):a quality of life instrument for use in clinical trials. Pract Diab Int 19:167-175.

5. Ribu L, Hanestad BR, Moum T, Birkeland K, Rustoen T (2007) Health related quality of life among patients with diabetes and foot ulcers: association with demographic and clinical characteristics. J Diabetes Complications 21: 227-236.

6. Ribu L, Birkeland K, Hanestad BR, Moum T, Rustoen T (2008) A longitudinal study of patients with diabetes and foot ulcers and their health-related quality of life: wound healing and quality-of-life changes. J Diabetes Complications 22: 400-407

7. Lipsky BA, Berendt AR, Deery HG, et al.; Infectious Diseases Society of America. Diagnosis and treatment of diabetic foot infections. Clin Infect Dis 2004;39:885-910

8. Jeffcoate WJ, Lipsky BA. Controversies in diagnosing and managing osteomyelitis of the foot in diabetes. Clin Infect Dis 2004;39(Suppl. 2):S115-S122

9. Lipsky BA, Berendt AR, Cornia PB, et al.; Infectious Diseases Society of America. 2012 Infectious Diseases Society of America clinical practice guideline for the diagnosis and treatment of diabetic foot infections. Clin Infect Dis 2012;54:e132-e173

10. Embil JM, Rose G, Trepman E, et al. Oral antimicrobial therapy for diabetic foot osteomyelitis. Foot Ankle Int. 2006;27:771-779

11. Senneville E, Lombart A, Beltrand E, et al. Outcome of diabetic foot osteomyelitis treated nonsurgical: a retrospective cohort study. Diabetes Care 2008;31:637-642

12. Game FL, Jeffcoate WJ. Primarily nonsurgical management of osteomyelitis of the foot in diabetes. Diabetologia 2008;51:962-967

\section{AJPHR is}

Peer-reviewed

monthly

Rapid publication

Submit your next manuscript at

editor@ajphr.com / editor.ajphr@gmail.com 